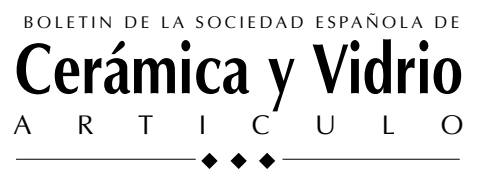

\title{
Estructura cristalina y propiedades eléctricas de soluciones sólidas $\mathrm{YCu}_{\mathrm{x}} \mathrm{Mn}_{1-\mathrm{x}} \mathrm{O}_{3}$
}

\author{
D. GUTIÉRREZ', C. MOURE', J. F. FERNÁNDEZ', O. PEÑA² \\ ${ }^{1}$ Instituto de Cerámica y Vidrio, CSIC, Departamento de Electrocerámica, 28500 Arganda, Madrid, España \\ ${ }^{2}$ Chimie du Solide et Inorganique Moléculaire (CSIM), UMR-CNRS 6511 Université de Rennes Cedex, France
}

\begin{abstract}
Se han estudiado las soluciones sólidas en el sistema $Y C u_{x} \mathrm{Mn}_{1-\mathrm{O}} \mathrm{O}_{3}$, entre 0 y $60 \%$ At. Cu. Los compuestos fueron preparados por reacción en estado sólido de los óxidos correspondientes. Los materiales cerámicos fueron sinterizados entre 1100 y $1300^{\circ} \mathrm{C}$. Con la incorporación a la manganita de itrio $\left(\mathrm{YMnO}_{3}\right)$ de cantidades superiores a $30 \%$ At. Cu, se produce la formación de una fase con estructura perovskita y simetría ortorrómbica. El aumento del contenido de Cu hasta una cantidad de $50 \%$ At., no afecta apreciablemente el factor de ortorrombicidad b/a. Para cantidades superiores a $50 \%$ At. Cu se obtiene un sistema multifásico, observándose $Y_{2} \mathrm{O}_{3}, \mathrm{MnnO}_{3}, \mathrm{Cu}_{2} Y_{2} \mathrm{O}_{5}$ además de la estructura perovskita. Las soluciones sólidas con estructura perovskita muestran un comportamiento eléctrico de material semiconductor, con valores de conductividad $(\sigma)$ que aumentan con el contenido de $\mathrm{Cu}$ hasta $\sim 33 \%$ At. $\mathrm{Cu}(\mathrm{x}=0.33)$, para luego decrecer hasta $\mathrm{x}=0.5$. La conductividad en estos materiales cerámicos está controlada por el mecanismo de salto activado de pequeño polarón. Los resultados se discuten en función de la razón $\mathrm{Mn}^{3+} / \mathrm{Mn}^{4+}$ para cada composición.
\end{abstract}

Palabras Clave: Manganitas, soluciones sólidas, propiedades eléctricas, cerámicas

Crystalline structure and electrical properties of $\mathrm{YCu}_{x} \mathrm{Mn}_{1-\mathrm{x}} \mathrm{O}_{3}$ solid solutions

Solid solutions belonging to the Mn-rich region of the $Y C u_{x} M n_{1-x} \mathrm{O}_{3}$ system have been studied. The powders were prepared by solid-state reaction between the corresponding oxides. Sintered ceramics were obtained by firing at $1100-1200^{\circ} \mathrm{C}$. The incorporation of 30 atomic $\% \mathrm{Cu}$ to the yttrium manganite induces the formation of a perovskite-type phase, with orthorhombic symmetry. Increase of the $\mathrm{Cu}$ amount do not appreciably affects the orthorhombicity factor $\mathrm{b} / \mathrm{a}$, up to an amount of 50 atomic \% Cu. Above this $\mathrm{Cu}$ amount, a multiphase system has been observed, with the presence of unreacted $\mathrm{Y}_{2} \mathrm{O}_{3}, \mathrm{YMnO}_{3^{\prime}} \mathrm{Cu}_{2} \mathrm{Y}_{2} \mathrm{O}_{5}$ and, the perovskite phase. DC electrical conductivity measurements have shown a semiconducting behavior for all the solid solutions with perovskite-type structure. The room temperature conductivity increases with $\mathrm{Cu}$ until $\sim 33$ atomic $\% \mathrm{Cu}$, and then decreases. Small polaron hopping mechanism, between $\mathrm{Mn}^{3+}$ and $\mathrm{Mn}^{4+}$ cations, controls the conductivity in these ceramics. Results are discussed as a function of the $\mathrm{Mn}^{3+} / \mathrm{Mn}^{4+}$ ratio for each composition.

Keywords: Manganites, perovskite, solid solutions, electrical properties, ceramics.

\section{INTRODUCCIÓN}

Las manganitas de Tierras Raras (TR) han sido objeto de gran interés debido a sus propiedades eléctricas (comportamiento semiconductor) y magnéticas (características magnetorresistivas). Se ha desarrollado un extenso trabajo sobre las propiedades de las manganitas de TR ligeras, particularmente sobre el conocimiento de las características del compuesto $\mathrm{LaMnO}{ }_{3}$, modificado mediante la sustitución de $\mathrm{Sr}^{2+} \mathrm{o} \mathrm{Ca}^{2+}$ por $\mathrm{La}^{3+}$ (1). La utilización de estas soluciones sólidas como electrodos cerámicos en pilas de combustible de óxidos sólidos (SOFC's) ha sido estudiada desde hace muchos años (2). Posteriormente, el efecto de magnetorresistencia colosal, encontrado tanto en cristales simples como en el material cerámico volumétrico de estas soluciones, ha sido ampliamente estudiado por muchos autores (3).

Las manganitas de TR ligeras cristalizan con una estructura de tipo perovskita y grupo espacial (G.E.) Pbnm, la cual tien- de a aumentar su anisotropía cuando el peso atómico de las TR aumenta y su radio iónico disminuye: desde la simetría romboédrica, cuasi-cúbica para $\mathrm{La}^{3+}$, a simetría ortorrómbica para $\mathrm{Dy}^{3+}$. Para iones como $\mathrm{Er}^{3+}$ y más pequeños, las manganitas de TR cristalizan con una simetría hexagonal y G.E. $\mathrm{P} 63 \mathrm{~cm}$ (4), a pesar de los valores correspondientes del factor de tolerancia de Goldschmidt:

$$
\mathrm{t}=\left(\mathrm{r}_{\mathrm{A}}+\mathrm{r}_{0}\right) /\left[\sqrt{2}\left(\mathrm{r}_{\mathrm{B}}+\mathrm{r}_{0}\right)\right]
$$

que define la estabilidad de la estructura. El cambio estructural a la simetría hexagonal puede ser asociado no solamente al decrecimiento del factor de tolerancia, sino también a la presencia del catión $\mathrm{Mn}^{3+}$, el cual es del tipo Jahn-Teller, en sitios B con coordinación octaédrica o menor. Este catión promueve una fuerte deformación anisotrópica, la cual induce el cam- 
bio de simetría, cuando el factor de tolerancia alcanza un valor suficientemente pequeño (5). Contrariamente, las ferritas de Tierras Raras pesadas, tal como $\mathrm{ErFeO}_{3}$ con igual factor de tolerancia que $\mathrm{ErFeO}_{3}(\sim 0.80)$, cristalizan con una estructura ortorrómbica de tipo perovskita.

El compuesto $\mathrm{YMnO}_{3}$, con estructura hexagonal, tiene propiedades de material ferroeléctrico y antiferromagnético con valores de conductividad eléctrica muy bajos (6). Para voltajes altos muestra un comportamiento no-óhmico peculiar (7). Por otra parte, las soluciones sólidas del tipo $\mathrm{Y}_{1-x} \mathrm{Ca}_{x} \mathrm{MnO} \mathrm{O}_{3}$ muestran una transición cristalina, de la estructura hexagonal a una estructura de tipo perovskita para cantidades de $\mathrm{Ca}$ mayores de $20 \%$ At. Estas soluciones son compuestos semiconductores, con energías de activación que caen a valores muy bajos con un aumento siguiente de la conductividad al aumentar el contenido de Ca (8). Este hecho hace posible el uso de las soluciones sólidas como electrodos cerámicos en celdas de combustible.

En la actualidad, existe poco trabajo sobre de la modificación de las manganitas de itrio por medio de la incorporación de los cationes apropiados en la subred del $\mathrm{Mn}^{3+}$ (9). En este trabajo se pretende estudiar el efecto de la incorporación de cationes $\mathrm{Cu}^{2+}$ (en sustitución de $\mathrm{Mn}^{3+}$ ) en el compuesto $\mathrm{YMnO}_{3}$ sobre el comportamiento de la estructura, la simetría y las propiedades eléctricas de las correspondientes soluciones sólidas $Y\left(\mathrm{Cu}_{x} \mathrm{M} n_{1-x}\right) \mathrm{O}_{3}$.

\section{PROCEDIMIENTO EXPERIMENTAL}

Se prepararon composiciones de $Y\left(\mathrm{Cu}_{x} \mathrm{Mn}_{1-x}\right) \mathrm{O}_{3}$ para $\mathrm{x}=0.2$ 0.6 , por reacción en estado sólido de la mezcla estequiométrica de los óxidos, $\mathrm{MnO}, \mathrm{CuO}$ e $\mathrm{Y}_{2} \mathrm{O}_{3}$-de tamaño de partícula submicrónico. Las mezclas fueron homogeneizadas mediante molienda en molino de atricción, utilizando bolas de circonio e isopropanol como medio líquido. Estas se sometieron a un proceso de secado a $60^{\circ} \mathrm{C}$, luego fueron tamizadas a través de una malla de $65 \mu \mathrm{m}$ y calcinadas a $1000{ }^{\circ} \mathrm{C}$ durante $1 \mathrm{~h}$. El producto calcinado fue molido utilizando la misma técnica, secado, granulado y prensado isostáticamente, para luego ser sinterizado.

La densidad aparente de las muestras sinterizadas fue determinada por desplazamiento en agua. El análisis de difracción de rayos X (XRD) fue realizado tanto en el polvo calcinado como en las muestras sinterizadas, utilizando un difractómetro D-5000 Siemens $(\mathrm{Cu} \mathrm{K} \alpha)$. El polvo fue identificado a una velocidad de barrido de $2^{\circ} 2 \theta / \mathrm{min}$. Los parámetros de red fueron calculados, para las muestras sinteri-

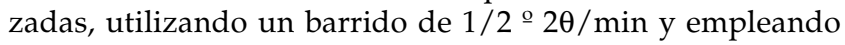
$\mathrm{Si}$ en polvo como estándar interno. La microestructura de las cerámicas sinterizadas fue observada mediante un Microscopio Electrónico de Barrido (SEM) sobre superficies pulidas, atacadas térmicamente y sobre superficies de fractura recientes.

Para la medida de la conductividad eléctrica se utilizaron muestras en forma de barras cilíndricas de $0.3 \mathrm{~cm}$ de diámetro por $1,5 \mathrm{~cm}$ de longitud, con pasta de plata como electrodo, que fue sinterizada a $775{ }^{\circ} \mathrm{C}$ durante $1 \mathrm{~h}$. Las medidas de conductividad fueron realizadas en el intervalo de temperatura de $25-700^{\circ} \mathrm{C}$, utilizando el método de cuatro puntas. Para las medidas se empleó una fuente Tektronix de corriente contínua, modelo PS280 y un multímetro HP modelo $34401 \mathrm{~A}$, con una resolución de $1 \mu \mathrm{A} \mathrm{DC}$, controlados mediante un ordenador.

\section{RESULTADOS Y DISCUSIÓN}

\subsection{Microestructura}

El tamaño aparente de partícula, medido por conteo laser fue de $\sim 1.3 \mu \mathrm{m}$; tamaño que corresponde principalmente a los agregados. Las medidas de superficie específica mediante la técnica BET, indican valores de $5 \mathrm{~m}^{2} / \mathrm{g}$, los cuales se correlacionan con un tamaño promedio de partícula de $\sim 0.2 \mu \mathrm{m}$.

Los polvos prensados y prerreaccionados fueron sinterizados en atmósfera de aire a diferentes temperaturas y tiempos. Se observaron las máximas densidades aparentes para muchos de los compuestos a $1150 \stackrel{\circ}{\circ} / 2 \mathrm{~h}$. Las densidades se encontraban dentro del rango 92-96\% de la densidad teórica.

La figura 1 describe la microestructura de las muestras sinterizadas. Puede observarse porosidad intragranular de tamaño pequeño y predominantemente la presencia de porosidad intergranular. La distribución mayoritaria de tamaño de grano se encuentra entre 1 y $3 \mu \mathrm{m}$ de diámetro.

La figura 2 muestra el análisis de difracción de rayos $\mathrm{X}$ de las muestras sinterizadas. Se observa para cantidades de $\mathrm{Cu}$ superiores a $30 \%$ At., la formación de una fase con características de la estructura perovskita. Para cantidades por encima de $50 \%$ At. $\mathrm{Cu}$ se aprecia un patrón diferente, observándose la presencia de $\mathrm{Y}_{2} \mathrm{O}_{3}$ sin reaccionar, $\mathrm{Cu}_{2} \mathrm{Y}_{2} \mathrm{O}_{5}$ e $\mathrm{YMnO}_{3}$.

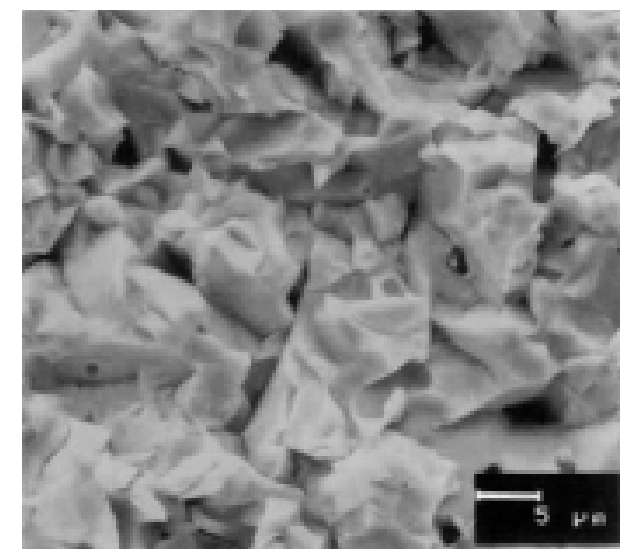

Figura 1. Micrografía MEB de una fractura de una muestra correspondiente a la composición con $33 \%$ At. Cu, sinterizada a $1150{ }^{\circ} \mathrm{C}, 2 \mathrm{~h}$.

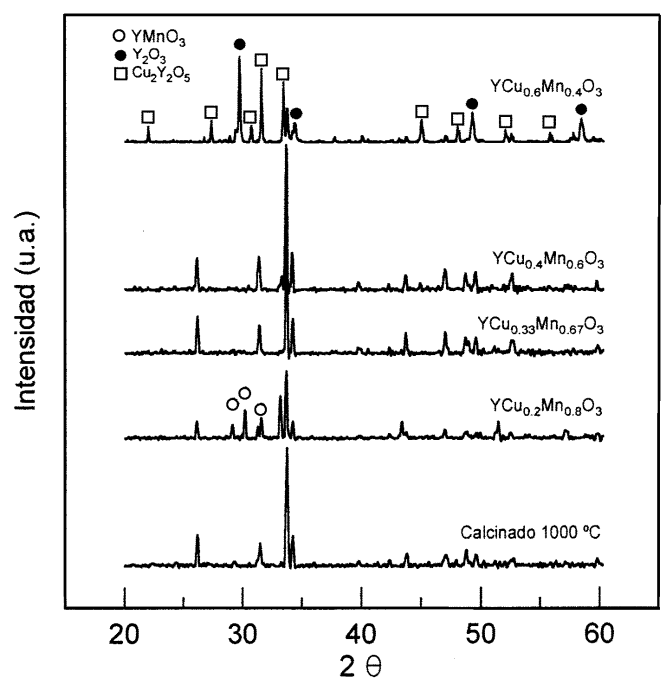

Figura 2: Espectros de Rayos X de las soluciones sólidas correspondientes a las muestras con $20 \%, 33 \%, 40 \%$ y $60 \%$ At. Cu. 
De acuerdo a lo observado (figura 2), la formación de la fase de estructura tipo perovskita aparece para cantidades entre 25 y $50 \%$ At. Cu. Para composiciones con una cantidad inferior a $30 \%$ At. $\mathrm{Cu}$, se observa la presencia de dos fases; la fase hexagonal principal $\mathrm{YnnO}_{3}$ y la fase de tipo perovskita, la cual crece con la concentración de $\mathrm{Cu}$. Para muestras con cantidades de 30 a $50 \%$ At. Cu, se identifica una única fase. Esta fase fue catalogada como un compuesto del tipo perovskita de simetría ortorrómbica, isomorfo al compuesto $\mathrm{GdMnO}_{3}$ y de grupo espacial Pbnm. Como se observa en la Tabla 1, la ortorrombicidad $(b / a)$ de la fase perovskita no experimenta cambios muy apreciables con la variación de la cantidad de $\mathrm{Cu}$.

En la figura 3 se observa el comportamiento de los valores medidos de los parámetos de red como una función del contenido de $\mathrm{Cu}$.

El comportamiento cristaloquímico de las muestras con cantidades de $\mathrm{Cu} \leq 50 \%$ At. es muy similar al observado en otros sistemas semejantes, como $(Y, \mathrm{Ca}) \mathrm{MnO}_{3} ;(\mathrm{Er}, \mathrm{Ca}) \mathrm{MnO}_{3}$; $(\mathrm{Gd}, \mathrm{Ca}) \mathrm{MnO}_{3}$ e $\mathrm{Y}(\mathrm{Ni}, \mathrm{Mn}) \mathrm{O}_{3}$. (10)

La sustitución del catión $\mathrm{Mn}^{3+}$ por un catión de menor valencia genera la aparición de $\mathrm{Mn}^{4+}$, en la cantidad correspondiente al $\mathrm{Mn}^{3+}$ sustituido (establecido por el equilibrio de carga). Aunque el catión $\mathrm{Cu}^{2+}$ sea del tipo Jahn-Teller, esta sustitutición disminuye la alta anisotropía de la red cristalina y promueve la aparición de la fase perovskita, indicando que el efecto Jahn-Teller es más significativo con la presencia de cationes $\mathrm{Mn}^{3+}$. Cuando se compara la ortorrombicidad de las soluciones con aquellas del compuesto $G d M n O_{3}(10)$, se puede apreciar que la razón b/a de todas las soluciones sólidas en estudio, es siempre menor a aquellas de la perovskita pura de $\mathrm{Gd}^{3+}$. Sin embargo, el factor de tolerancia de estas estructuras es menor que el de la perovskita de $\mathrm{Gd}^{3+}$ mencionada. Para las soluciones sólidas $(Y, \mathrm{Ca}) \mathrm{MnO}_{3}$, el factor de tolerancia aumenta con el contenido de $\mathrm{Ca}^{2+}$, debido al mayor radio iónico del catión comparado al del $\mathrm{Y}^{3+}$, el cual lleva a un aumento del radio iónico medio en las posiciones A. Contrariamente, en las soluciones sólidas del presente caso, el factor de tolerancia $(t)$ permanece prácticamente sin cambio alguno. No obstante, el radio iónico del $\mathrm{Cu}^{2+}$ es algo mayor al del $\mathrm{Mn}^{3+}$, por lo que esto podría causar una disminución en el valor de $t$; esta disminución puede ser compensada, si se supone que parte de los cationes de $\mathrm{Mn}^{3+}$ están cambiando al estado de valencia $\mathrm{Mn}^{4+}$, con un radio iónico inferior. Por lo tanto, la razón del cambio al tipo de estructura perovskita puede ser principalmente atribuida a la desaparición progresiva de los cationes Jahn-Teller $\mathrm{Mn}^{3+}$ y no a la modificación del factor de tolerancia. Lo cambios leves en el valor de $t$ podrían ser causa del comportamiento de la razón $b / a$, con una pequeña reducción para valores entre 25 y $33 \%$ At. y muy poca variación desde 33 a $50 \%$ At.

Los valores bajos de $t$ favorecen el alto grado de asimetría de la red. El grado de compactación de la red perovskita aumenta, incrementándose también la densidad cristalográfica con el contenido de $\mathrm{Cu}^{2+}$.

Comparando los resultados obtenidos con otros sistemas estudiados, como los anteriormente citados: $(Y, \mathrm{Ca}) \mathrm{MnO}_{3}$, $(\mathrm{Er}, \mathrm{Ca}) \mathrm{MnO}_{3}$ e $\mathrm{Y}(\mathrm{Ni}, \mathrm{Mn}) \mathrm{O}_{3}$ (10), se puede establecer que el cambio de la estructura hexagonal a la de tipo perovskita se da prácticamente a la misma concentración del catión modificador, es decir, cuando la cantidad de $\mathrm{Mn}^{3+}$ disminuye hasta un valor crítico alrededor de $75 \%$ At. de la cantidad original, independientemente del valor del factor de tolerancia correspondiente, estabilizando la red perovskita.
TABla 1. PARÁmetros DE RED, CONDUCTIVIDAD ELÉCTRICA $\left(25^{\circ} \mathrm{C}\right)$ Y ENERGÍA DE ACTIVACIÓN PARA LAS SOLUCIONES DE TIPO PEROVSKITA COMO UNA FUNCIÓN DEL CONTENIDO DE CU.

\begin{tabular}{|c|c|c|c|c|c|c|}
\hline At. \% Cu & 20 & 30 & 33 & 40 & 45 & 50 \\
\hline $\mathrm{a}$ & 5.2457 & 5.2531 & 5.2545 & 5.2472 & 5.2470 & 5.2460 \\
\hline $\mathrm{b}$ & 5.7297 & 5.7193 & 5,7153 & 5,7184 & 5,7138 & 5.7215 \\
\hline $\mathrm{c}$ & 7.3816 & 7.3631 & 7.3618 & 7.3671 & 7.3713 & 7.3682 \\
\hline $\mathrm{b} / \mathrm{a}$ & 1.092 & 1.089 & 1.088 & 1.090 & 1.089 & 1.091 \\
\hline $\mathrm{V}\left[\mathrm{nm}^{3}\right]$ & 0.2219 & 0.2212 & 0.2211 & 0.2211 & 0.2210 & 0.2212 \\
\hline$\sigma\left[\mathrm{Sm}^{-1}\right]$ & - & 0.104 & 0.119 & - & 0.072 & 0.039 \\
\hline $\mathrm{E}_{\text {activ }}[\mathrm{eV}]$ & - & 0.26 & 0.24 & 0.27 & 0.22 & 0.26 \\
\hline
\end{tabular}

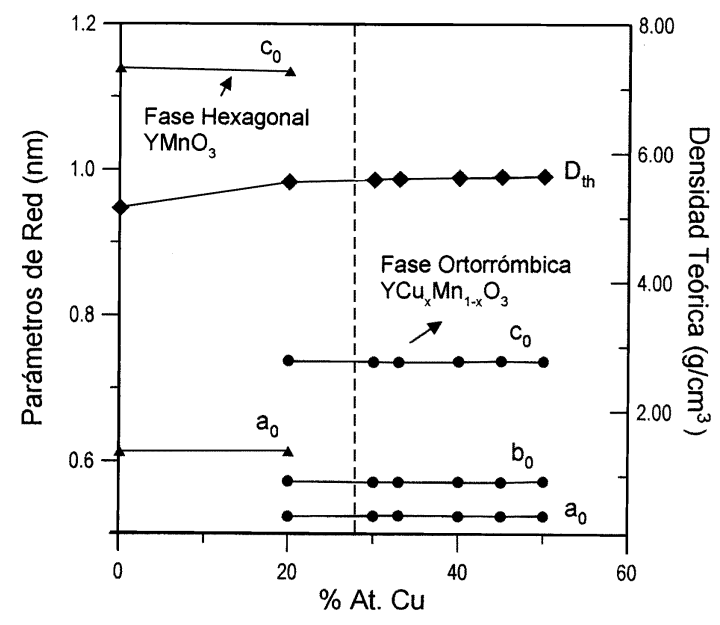

Figura 3: Variación de los parámetros de red con la concentración de Cu.

\subsection{Conductividad eléctrica}

En la figura 4 se observa el comportamiento eléctrico, como una función de la concentración de $\mathrm{Cu}$, en el intervalo de 300 a $700 \mathrm{~K}$. Se observa un incremento de la conductividad hasta una concentración de $\mathrm{Cu}$ de 33 \% At., luego decrece gradualmente con la concentración. Para temperaturas superiores a $500 \mathrm{~K}$, se observa un incremento de la conductividad para el intervalo de concentración de 45-50\% At. Cu. El comportamiento de crecimiento y decrecimiento, con un valor máximo en $x=0.33$, puede explicarse a partir de la relación de concentración de cationes $\mathrm{Mn}^{3+} / \mathrm{Mn}^{4+}$. Es posible formar 0.33 pares $\mathrm{Mn}^{3+}-\mathrm{Mn}^{4+}$ por unidad de fórmula en la composición 33/67 $([\mathrm{Cu}] /[\mathrm{Mn}])$, mientras es posible formar solamente 0.2 y 0.1 en las composiciones $40 / 60$ y $45 / 55$, respectivamente.

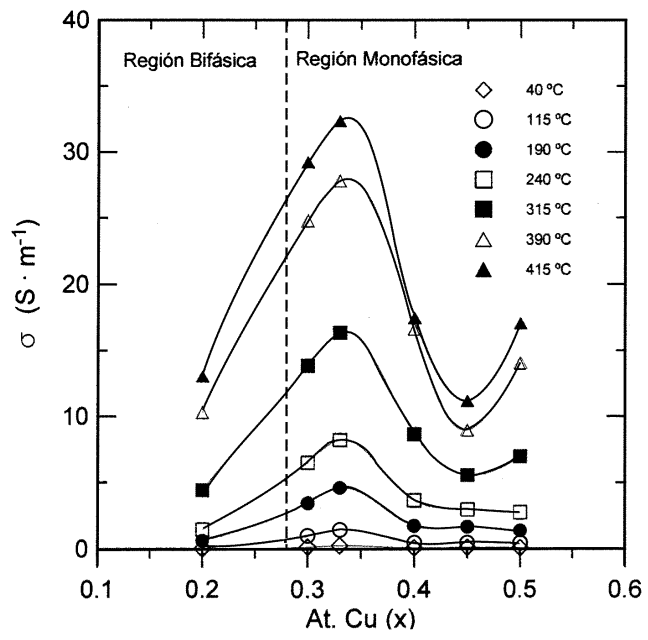

Figura 4: Comportamiento de la conductividad eléctrica con la concentración de $\mathrm{Cu}$ y la temperatura. 
Esta variación en el porcentaje relativo entre los dos cationes de Mn explica la existencia de un máximo en la conductividad para valores intermedios de la concentración de $\mathrm{Cu}$. El aumento de la conductividad a temperaturas superiores, en el intervalo $0.33-0.50$, podría estar relacionado con la naturaleza del catión sustituyente, es decir, con la interacción entre cationes y los diferentes estados de valencia, dando como resultado un cambio de la simetría hacia una simetría de orden superior y un consecuente cambio en la energía de activación (9). Aún se requiere trabajo adicional para probar la validez de esta hipótesis.

Del comportamiento lineal de las curvas $\log \sigma$ y $\log \sigma T$ versus $1 / T$ observado en la figuras $5 a-5 b$, se puede establecer que el mecanismo de conducción para estas soluciones sólidas es por pequeño polarón térmicamente activado, similarmente a otros semiconductores de valencia controlada, como en las soluciones sólidas $\mathrm{La}_{1-x} \mathrm{Sr}_{x} \mathrm{MnO}_{3}$ (11).

En la Tabla I se reúnen los valores de conductividad a temperatura ambiente y la energía de activación para la conducción eléctrica de las muestras monofásicas, calculada a partir de las correspondientes curvas de Arhenius $\ln \sigma-1 / T$ para el intervalo de temperatura de medida. A la temperatura de 300 $\mathrm{K}$, las soluciones sólidas $Y \mathrm{C} u_{0.40} \mathrm{Mn}_{0.60} \mathrm{O}_{3}$ presentan un valor de $\mathrm{B} \sim 3200 \mathrm{~K}$ (constante relacionada con el modelo de conducción en los resistores con coeficiente de temperatura negativo (12)), calculada a partir de la inversa de la pendiente de la curva $\ln \sigma-1 / T$, con un coeficiente de temperatura del resistor ("TCR") $\alpha_{\mathrm{R}} \sim-4 \% \mathrm{~K}^{-1}$, el cual es comparable al de algunos termistores comerciales.

\section{CONCLUSIONES}

La sustitución de $\mathrm{Mn}^{3+}$ por $\mathrm{Cu}^{2+}$ (ambos iones del tipo Jahn-Teller) en el compuesto hexagonal $\mathrm{YMnO}_{3}$ induce una transición de una fase hexagonal a una ortorrómbica, de tipo perovskita, para cantidades de $\mathrm{Cu}$ entre 25 y $50 \%$ At., en forma similar a la reportada para soluciones sólidas $(\mathrm{Y}, \mathrm{Ca}) \mathrm{MnO}_{3}$ e $\mathrm{Y}(\mathrm{Ni}, \mathrm{Mn}) \mathrm{O}_{3}$. La causa de esta transición está relacionada con la disminución de los cationes $\mathrm{Mn}^{3+}$ en la red.

Para concentraciones de $\mathrm{Cu}$ menores de $0.3 \%$ At., se observa la fase $\mathrm{YMnO}_{3}$, coexistiendo con la fase perovskita $Y(\mathrm{Cu}, \mathrm{Mn}) \mathrm{O}_{3}$. Por encima de $50 \%$ At. $\mathrm{Cu}$, se ha observado una región multifásica en la que aparece además de la fase perovskita, $\mathrm{Y}_{2} \mathrm{O}_{3}$ sin reaccionar, $\mathrm{Cu}_{2} \mathrm{Y}_{2} \mathrm{O}_{5}$ e $Y M n \mathrm{O}_{3}$.

Las soluciones sólidas $\mathrm{Y}(\mathrm{Cu}, \mathrm{Mn}) \mathrm{O}_{3}$ de tipo perovskita, son materiales semiconductores, en las que el mecanismo de conducción mayoritario se debe al salto activado térmicamente de pequeño polarón entre los cationes $\mathrm{Mn}^{3+}-\mathrm{Mn}^{4+}$. Los materiales presentan buenos valores de conductividad eléctrica, para una posible utilización como electrodos cerámicos para pilas de combustible (SOFC'S), además de la posibilidad de su utilización como materiales termistores.

\section{AGRADECIMIENTOS}

Los autores agradecen al Ministerio de Ciencia de España el apoyo recibido a través del proyecto CICYT-MAT-97-0679 -C02-01.

\section{BIBLIOGRAFÍA}

1. A. Hammouche, E. Siebert, A. Hammou. "Crystallographic, thermal and electrochemical properties of the system $\mathrm{La}_{1-x} \mathrm{Sr}_{x} \mathrm{MnO}_{3}$ for high temperature Solid
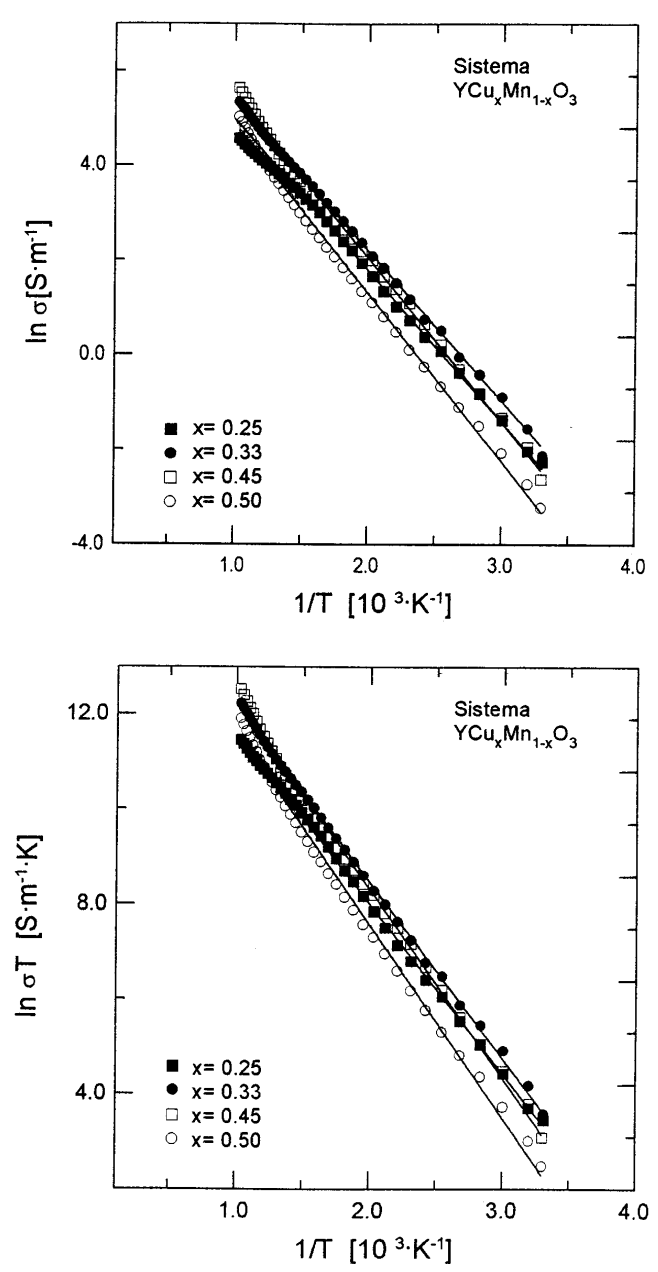

Figura 5: a) $\ln \sigma$ versus $1 / T$ b) $\ln \sigma T$ versus $1 / T$

Oxide Fuel Cell". Mater., Res. Bull, 24 367-80 (1989).

2. J. Tanaka, K. Takahashi, K. Yukino, S. Horiuchi. "Electrical conduction of $\left(\mathrm{La}_{0.80} \mathrm{Ca}_{0.20}\right) \mathrm{MnO}_{3}$ with homogeneous ionic distribution". Phys. Status Solidi, $80621-630$ (1983).

3. M. Ostergar, M .J .L \& Mogensen. "AC impedance study of the oxygen reduction mechanism on $\mathrm{La}_{x} \mathrm{Sr}_{1-x} \mathrm{MnO}_{3}$ in SOFC". Electrochimica Acta 38 2015-2020 (1993).

4. Y. Urishabara, A. Moritomo, Y. Arima, T. Asatmisu, A. Kido, G \& Yokura. "Insulator-metal transition and giant magnetoresistance in $\mathrm{La}_{1-\mathrm{x}} \mathrm{Sr}_{\mathrm{x}} \mathrm{MnO}_{3}$. Phys. Rew. B 14 103-114 (1995-II).

5. O. Muller, R.Roy. "The Major Ternary Structural Families". New York: Springer Verlag, 357-358 (1974).

6. H. L. Yakel, W.C. Koehler, E.F. Berataut, E. F. Forrat. "On the Crystal Structure of the Manganese (III) Trioxides of the Heavy Lanthanides and Yttrium", Acta Crys. 16957 (1963).

7. C. Moure, J.F. Fernández, M. Villegas, P. Durán. "Non-Ohmic Behavior and Switching phenomena in $\mathrm{YMnO}_{3}$ based ceramic materials". J. European Cer. Soc. 19 131-137 (1999).

8. C. Moure, M. Villegas, J.F. Fernández, P. Durán. "Phase transition and electrical conductivity in the system $\mathrm{YMnO}_{3}-\mathrm{CaMnO}_{3}$ ". J. Mater. Sci. 34 2565-2568 (1999).

9. D. Gutiérrez, J. F. Fernández, P. Durán, C. Moure. "Crystalline structure and electrical properties of solid solutions $\mathrm{YNi}_{x} \mathrm{Mn}_{1-\mathrm{x}} \mathrm{O}_{3}$ ". Bol. Soc. Esp. Cerám. Vidrio 38 [6] 653-657 (1999).

10. C. Moure, D. Gutiérrez, J.F. Fernández, J. Tartaj, P. Durán, O. Pena. "Phase transitions induced on hexagonal manganites by the incorporation of aliovalent cations on A or B sites". Bol. Soc. Esp. Cerám. Vidrio 38 [5] 417-420 (1999).

11. G. Subba, B. Wanklyn, C.N. Rao. "Electrical Transport in Rare-Earth OrthoChromites, Manganites and Ferrites". J. Phys. Chem. Solids 32 345-358 (1971).

12. A.J. Moulson, J. M. Herbet. "Electroceramics, Materials Properties and Aplications". Cambridge: Chapman \& Hall, 139-154 (1990). 